\title{
Fenologia de floração e polinização de espécies ornitófilas de bromeliáceas em uma área de campo rupestre da Chapada Diamantina, BA, Brasil ${ }^{1}$ CYRIO SILVEIRA SANTANA ${ }^{2,3}$ e CAIO GRACO MACHADO ${ }^{2}$
}

(recebido: 04 de maio de 2009; aceito: 12 de agosto de 2010)

\begin{abstract}
Flowering phenology and pollination of ornitophilous bromeliads in the campo rupestre of Chapada Diamantina, Bahia, Brazil). Hummingbirds are the major pollinators of bromeliads and the sequential flowering can potentially provide the maintenance of their local populations. In this study we investigated the phenological flowering strategies and the floral visitors of five ornitophilous bromeliads on the high altitude rocky field (campos rupestres) areas of the Chapada Diamantina, Bahia. Flowering phenology data were collected in monthly monitored individuals, from July 2006 to December 2007. Floral visitors were recorded by naturalistic observations from February 2002 to December 2003 and from July 2006 to December 2007. Most species flowered at the end of the dry season and the beginning of the rainy season, but Hohenbergia ramageana Mez showed continuous flowering. The community showed a sequential and continuous flowering, providing feeding resources for the maintenance of pollinators in the area throughout the year. Six hummingbirds, the perching bird, and three species of bees visited the bromeliads flowers. Chlorostilbon lucidus (Shaw, 1812), Phaethornis pretrei (Lesson \& Delattre, 1839), and Coereba flaveola (Linnaeus, 1759) were their major pollinators. Neoregelia bahiana (Ule) L. B. Sm., with its long corolla tube is the most specialized species, and P. pretrei was its only pollinator, while H. ramageana, with its short corolla tube is the most generalist, considered an important resource for the hummingbirds of the area.
\end{abstract}

Key words - bromeliads, Espinhaço Range, hummingbirds, ornithophily

RESUMO - (Fenologia de floração e polinização de espécies ornitófilas de bromeliáceas em uma área de campo rupestre da Chapada Diamantina, BA, Brasil). Beija-flores são os principais polinizadores de bromeliáceas e a floração sequencial propicia a manutenção local destas aves. Neste estudo investigamos as estratégias fenológicas de floração e os visitantes florais de cinco bromeliáceas ornitófilas em uma área de campo rupestre na Chapada Diamantina, Bahia. Os dados sobre fenologia de floração foram coletados pelo acompanhamento mensal da fenofase de floração de indivíduos no período entre julho de 2006 e dezembro de 2007. Os visitantes florais foram registrados em observações naturalísticas de fevereiro de 2002 a dezembro de 2003 e de julho de 2006 a dezembro de 2007. A maioria das espécies floresceu no fim da estação seca e no início da estação chuvosa; Hohenbergia ramageana Mez apresentou floração contínua na área. A comunidade de bromélias estudada apresentou floração seqüencial e contínua, proporcionando recursos para a manutenção dos polinizadores na área ao longo do ano. Seis troquilídeos, um cerebídeo e três espécies de abelhas visitaram flores das bromélias. Chlorostilbon lucidus (Shaw, 1812), Phaethornis pretrei (Lesson \& Delattre, 1839) e Coereba flaveola (Linnaeus, 1759) foram os principais polinizadores das plantas na área. Neoregelia bahiana (Ule) L. B. Sm., de corola de tubo longo é a espécie mais especializada, tendo P. pretrei como seu único polinizador, enquanto $H$. ramageana, com corola de tubo curto, é a mais generalista, considerada como um importante recurso para os beija-flores da área

Palavras-chave - beija-flores, bromélias, Cadeia do Espinhaço, ornitofilia

\section{Introdução}

$\mathrm{Na}$ família Bromeliaceae, com 56 gêneros e cerca de 3.000 espécies (Luther 2004), das quais aproximadamente $40 \%$ ocorrem no Brasil (Reitz 1983), a ornitofilia é a síndrome de polinização mais frequente

1. Parte da dissertação de mestrado do primeiro autor, Programa de Pós-Graduação em Botânica, Universidade Estadual de Feira de Santana, BA, Brasil.

2. Universidade Estadual de Feira de Santana - Departamento de Ciências Biológicas, Laboratório de Ornitologia (Ornito), Avenida Transnordestina s/n, Bairro Novo Horizonte, 44.036-900 Feira de Santana, BA, Brasil.

3. Autor para correspondência: cyrio_s@yahoo.com.br
(Martinelli 1997, Machado \& Semir 2006). Bromeliaceae é citada por vários autores como sendo responsável pelo maior percentual de espécies ornitófilas em diferentes comunidades vegetacionais (Snow \& Snow 1986, Buzato et al. 2000). Os beija-flores (Aves: Trochilidae) são considerados os principais agentes polinizadores de bromeliáceas (Alves et al. 2000, Siqueira Filho \& Machado 2001, Kaehler et al. 2005, Machado \& Semir 2006) e suas flores podem ser agrupadas em duas classes: especializadas, com corolas alongadas e generalistas, com corolas mais curtas (Fraga 1989).

Questões sobre a fenologia de floração de bromeliáceas têm sido relativamente pouco investigadas. A maioria dos estudos se concentra em regiões da Mata 
Atlântica, no sudeste brasileiro, apresentando uma tendência para a floração sequencial em comunidades de bromélias (Araujo et al. 2004, Machado \& Semir 2006, Piacentini \& Varassin 2007). No nordeste do Brasil há poucos estudos realizados (Siqueira Filho 1998, Siqueira Filho \& Machado 2001, Leal et al. 2006), sendo que na Chapada Diamantina algumas informações sobre fenologia de bromélias foram reportadas (Machado et al. 2007, Machado 2009).

O conhecimento do padrão fenológico de uma comunidade vegetal, assim como o seu sistema de polinização, é essencial para o entendimento das relações entre as plantas e os animais que dependem delas (Morellato \& Leitão-Filho 1992, Talora \& Morellato 2000). Este conhecimento é fundamental para a conservação dos ecossistemas naturais, contribuindo para o manejo e conservação de espécies endêmicas ou ameaçadas de extinção (Silva 1998), uma vez que as bromélias são potencializadoras da biodiversidade proporcionando a formação de reservatórios de água e nutrientes que tem um papel importante tanto para a nutrição das próprias plantas, como em constituir um micro ambiente onde vivem diversos invertebrados e vertebrados (Reitz 1983, Santos et al. 2003, Rocha et al. 2004). A Chapada Diamantina é considerada a mais importante área de endemismo de Bromeliaceae, abrigando $62 \%$ de todos os taxa endêmicos (Versieux \& Wendt 2007).

Este estudo objetivou investigar as estratégias fenológicas de floração, relacionar dados abióticos com a fenologia, investigar a biologia floral e comparar a sua morfologia com o bico dos beija-flores e determinar quais os visitantes florais da comunidade de bromeliáceas ornitófilas em uma área de campo rupestre da Chapada Diamantina, BA. Nesse contexto foi avaliado se as bromélias apresentaram floração contínua e sequencial e se os beija-flores foram seus principais polinizadores.

\section{Material e métodos}

Área de estudo - Este estudo foi realizado no Parque Municipal de Mucugê (PMM), em Mucugê, BA. O PMM situa-se a $12^{\circ} 59^{\prime} 18^{\prime \prime} \mathrm{S}$ e $41^{\circ} 20^{\prime} 27^{\prime \prime} \mathrm{W}$, possui uma área de 450 ha e está localizado a uma altitude a cerca de $950 \mathrm{~m}$. O clima é caracterizado como semiúmido, com estações chuvosa (outubro a março) e seca (abril a setembro). A pluviosidade média anual é de $1.100 \mathrm{~mm}$, com média de $600 \mathrm{~mm}$ na estação seca e $1.500 \mathrm{~mm}$ na estação chuvosa. A temperatura média anual é de cerca de $20^{\circ} \mathrm{C}$, com mínima de $13{ }^{\circ} \mathrm{C}$ na estação seca e máxima de $30^{\circ} \mathrm{C}$ na estação chuvosa (Stradmann 1998, Faustino \& Machado 2006).
A vegetação predominante no PMM é de campo rupestre (Stradmann 1998). Essa formação vegetal é encontrada em afloramentos rochosos com altitude acima de $900 \mathrm{~m}$, sendo muito comum em toda a Chapada Diamantina (Giulietti et al. 1997). Os estratos herbáceo e arbustivo são predominantes, formando um rico mosaico devido principalmente ao solo pouco profundo e arenoso, à natureza do substrato, à topografia e aos microclimas (Harley \& Simmons 1986, Giulietti et al. 1997, Conceição \& Giulietti 2002, Conceição \& Pirani 2005).

Espécies estudadas - Plantas ornitófilas são aquelas que apresentam flores de antese diurna, tubulosas, inodoras, com cores vistosas (ou em suas estruturas anexas), baixa concentração de açúcares (entre 20 e $30 \%$ ) e alta produção de néctar (Faegri \& van der Pijl 1979). Dentre as bromeliáceas que ocorrem no PMM, cinco são ornitófilas e foram selecionadas para este estudo: Aechmea bromeliifolia (Rudge) Baker, Hohenbergia ramageana Mez, Neoregelia bahiana (Ule) L. B. Sm., Orthophytum albopictum Philcox e O. mucugense Wand. \& A. A. Conc.

Todas essas espécies são rupícolas, exceto A. bromeliifolia que é encontrada em substrato arenoso e, assim como $O$. mucugense, são as únicas espécies esciófilas, enquanto que as demais são heliófilas. Orthophytum mucugense é a única espécie que ocorre em paredões úmidos às margens de rio. Hohenbergia ramageana é uma espécie considerada vulnerável na porção mineira da Cadeia do Espinhaço (Versieux \& Wendt 2007) e os gêneros Hohenbergia, Aechmea e Neoregelia como sendo característicos de Mata Atlântica. Orthophytum mucugense é também considerada vulnerável por ter sua distribuição conhecida apenas em Mucugê (Wanderley \& Conceição 2006). Estas cinco espécies pertencem à subfamília Bromelioideae, que tem frutos carnosos e zoocóricos, dentre outras características (Benzing 1980).

Coleta de dados - Os dados mensais sobre a precipitação acumulada e temperatura média foram coletados na estação climatológica da Fazenda Agropecuária São João do Paraguaçu, em Mucugê, a $20 \mathrm{~km}$ do PMM, e os dados de fotoperíodo foram calculados a partir das coordenadas geográficas do PMM, usando o Photoperiod Calculator (Lammi 2001), para serem correlacionados com a fenologia das bromélias ornitófilas do PMM.

Os dados sobre a fenofase de floração foram amostrados mensalmente no período de julho de 2006 a dezembro de 2007, totalizando 18 expedições à área de estudo, com duração média de cinco dias cada uma. Considerou-se a presença ou ausência de flores no PMM para a classificação da fenofase de floração de cada espécie estudada. Para a determinação do índice de atividade de floração e os registros do número de indivíduos com flores e do número de flores abertas por indivíduo/dia foram marcadas, ao acaso, com etiquetas plásticas, 50 inflorescências de cada espécie, com exceção de A. bromeliifolia e $N$. bahiana, com 12 e 26 inflorescências respectivamente, devido ao reduzido número de espécimes. 
Nos indivíduos com mais de uma roseta considerou-se apenas uma inflorescência.

Os padrões fenológicos de floração foram determinados considerando-se os critérios de frequência e duração(Newstron et al. 1994). Para determinar o índice de atividade de floração das plantas, foi registrada mensalmente a porcentagem de indivíduos floridos para cada espécie (Bencke \& Morellato 2002); quanto à sincronia de floração foram reconhecidas as seguintes categorias: assincrônica $(<20 \%$ dos indivíduos floridos), sincronia baixa (20 a 60\% dos indivíduos floridos) e sincronia alta ( $>60 \%$ dos indivíduos floridos) (Bencke \& Morellato 2002).

Para cada espécie foram registrados, em indivíduos aleatórios: o período de antese $(n=10)$, da abertura à senescência da flor, a cor da corola e das estruturas anexas (brácteas, escapo e folhas, por exemplo), o comprimento do tubo floral $(n=10)$, com o auxílio de paquímetro, a concentração de açúcares no néctar $(n=10)$, com o uso de microsseringa graduada para extrair o néctar e um refratômetro de bolso para medição da concentração, a presença de grãos de pólen nas anteras e a receptividade do estigma, medida a cada hora durante o período de antese $(n=10)$ (Dafni 1992).

O registro dos visitantes florais foi realizado em duas etapas: na primeira etapa foram seis visitas bimestrais de fevereiro a dezembro de 2002 e doze mensais, de janeiro a dezembro de 2003; a segunda etapa foi no período de julho de 2006 a dezembro de 2007. Todas as expedições tiveram uma duração média de cinco dias. Para determinar quais os visitantes florais das bromélias estudadas, foram realizadas sessões de observações naturalísticas, utilizando-se o método do indivíduo-focal (Altmann 1974), a olho desarmado ou com auxílio de binóculo $8 \times 30 \mathrm{~mm}$. Sempre que possível foram monitoradas mais de uma planta da mesma ou de outras espécies e as sessões se iniciavam na aurora e finalizavam-se quando as flores estavam completamente fechadas (Machado e
Rocca 2008). Durante esse período registraram-se as espécies visitantes e o tipo de visitas que realizavam, considerando como visitas legítimas as que o visitante contactava os órgãos reprodutivos das plantas.

As aves foram identificadas no campo com o auxílio de guia de identificação (Grantsau 1988) e a classificação seguiu CBRO (2007). As medidas do bico dos beija-flores foram obtidas de Grantsau (1988) e Vasconcelos \& Lombardi (2001) para correlacionar com o comprimento do tubo das corolas das plantas. Abelhas visitantes foram coletadas com redes entomológicas, fixadas em álcool 70\% e, assim como as bromélias, identificadas por especialistas.

Utilizou-se o teste de Shapiro e Wilk (Zar 1996) para verificar a normalidade da distribuição dos dados. Como estes não apresentaram distribuição normal foi utilizada a correlação de Spearman para avaliar se o número total de flores produzidas a cada mês apresentou relação com a pluviosidade, fotoperíodo e temperatura e se o comprimento dos tubos florais apresentou relação com o tamanho dos bicos dos beija-flores e com as concentrações do néctar das diferentes espécies (San Martin-Gajardo \& Morellato 2003).

\section{Resultados}

A maior parte das espécies de bromélias ornitófilas floresceu nos meses de setembro e outubro, período que corresponde ao fim da estação seca e início da estação chuvosa (figura 1). Em seu conjunto, a comunidade de bromeliáceas ornitófilas do PMM apresentou padrão de floração sequencial e contínuo (figura 1).

Hohenbergia ramageana apresentou padrão de floração do tipo contínuo, sendo a única espécie que floresceu durante todo o ano (figura 1), com uma alta

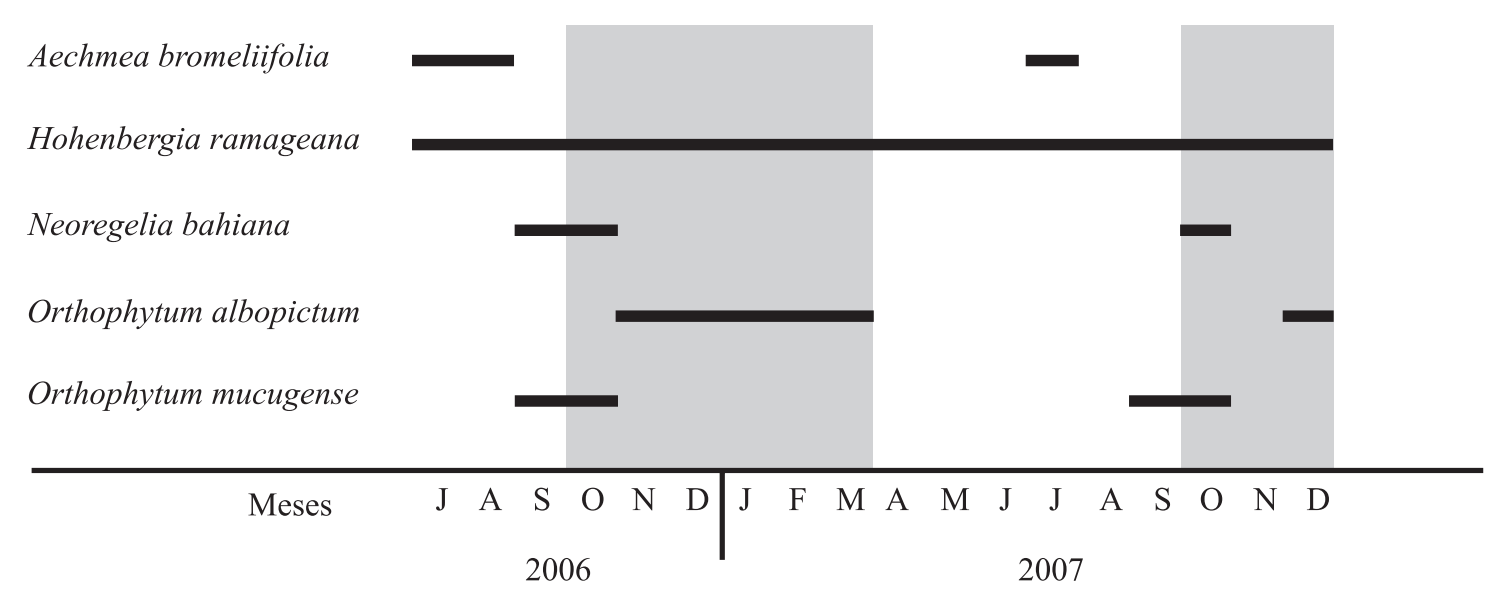

Figura 1. Período da floração das espécies de bromélias ornitófilas no Parque Municipal de Mucugê, Mucugê, Bahia, de julho de 2006 a dezembro de 2007. Áreas sombreadas representam a estação chuvosa e as claras a estação seca.

Figure 1. Flowering period of ornithophilous species of bromeliads in the Mucugê municipal park, Mucugê, Bahia, from July 2006 to December 2007. Shaded areas represent the rainy season, and the clear ones the dry season. 
sincronia na maior parte deste período e com um maior número de indivíduos florindo na estação seca (figura 2). Neoregelia bahiana e Orthophytum mucugense floresceram no fim da estação seca e início da chuvosa. Aechmea bromeliifolia floresceu na estação seca, enquanto Orthophytum albopictum na chuvosa (figura 1). Estas espécies apresentaram frequência de floração anual com duração intermediária (figura 1). As florações das espécies de Orthophytum foram assincrônicas, enquanto $A$. bromeliifolia e $N$. bahiana alternaram em baixa sincronia e assincronia (figura 2).

Houve correlação negativa entre a média de flores/ mês dos indivíduos marcados de $H$. ramageana e a pluviosidade $\left(\mathrm{r}_{\mathrm{s}}=-0,480, P<0,05\right)$ e correlação positiva entre a média de flores/mês dos indivíduos marcados de
O. albopictum e a pluviosidade $\left(\mathrm{r}_{\mathrm{S}}=0,552, P<0,05\right)$. Aechmea bromeliifolia e $H$. ramageana apresentaram correlação negativa entre a média de flores/mês dos indivíduos marcados e o fotoperíodo (respectivamente $\mathrm{r}_{\mathrm{S}}=-0,516, P<0,05$ e $\left.\mathrm{r}_{\mathrm{S}}=-0,516, P<0,05\right)$ e a média de flores/mês dos indivíduos marcados e a temperatura $\left(\mathrm{r}_{\mathrm{S}}=-0,552, \quad P<0,05 \quad\right.$ e $\quad \mathrm{r}_{\mathrm{S}}=-0,632, \quad P<0,05$, respectivamente).

A floração de $A$. bromeliifolia, $N$. bahiana e $O$. mucugense não apresentou correlação com a pluviosidade $\left(\mathrm{r}_{\mathrm{S}}=-0,238, P>0,05 ; \mathrm{r}_{\mathrm{S}}=-0,135, P>0,05 ; \mathrm{r}_{\mathrm{S}}=0,148\right.$, $P>0,05)$, assim como N. bahiana, O. albopictum e $O$. mucugense não apresentou correlação com fotoperíodo $\left(\mathrm{r}_{\mathrm{S}}=0,108, P>0,05 ; \mathrm{r}_{\mathrm{S}}=0,339, P>0,05 ; \mathrm{r}_{\mathrm{s}}=0,115\right.$, $P>0,05)$ e temperatura $\left(\mathrm{r}_{\mathrm{S}}=-0,006, P>0,05 ; \mathrm{r}_{\mathrm{S}}=0,263\right.$,
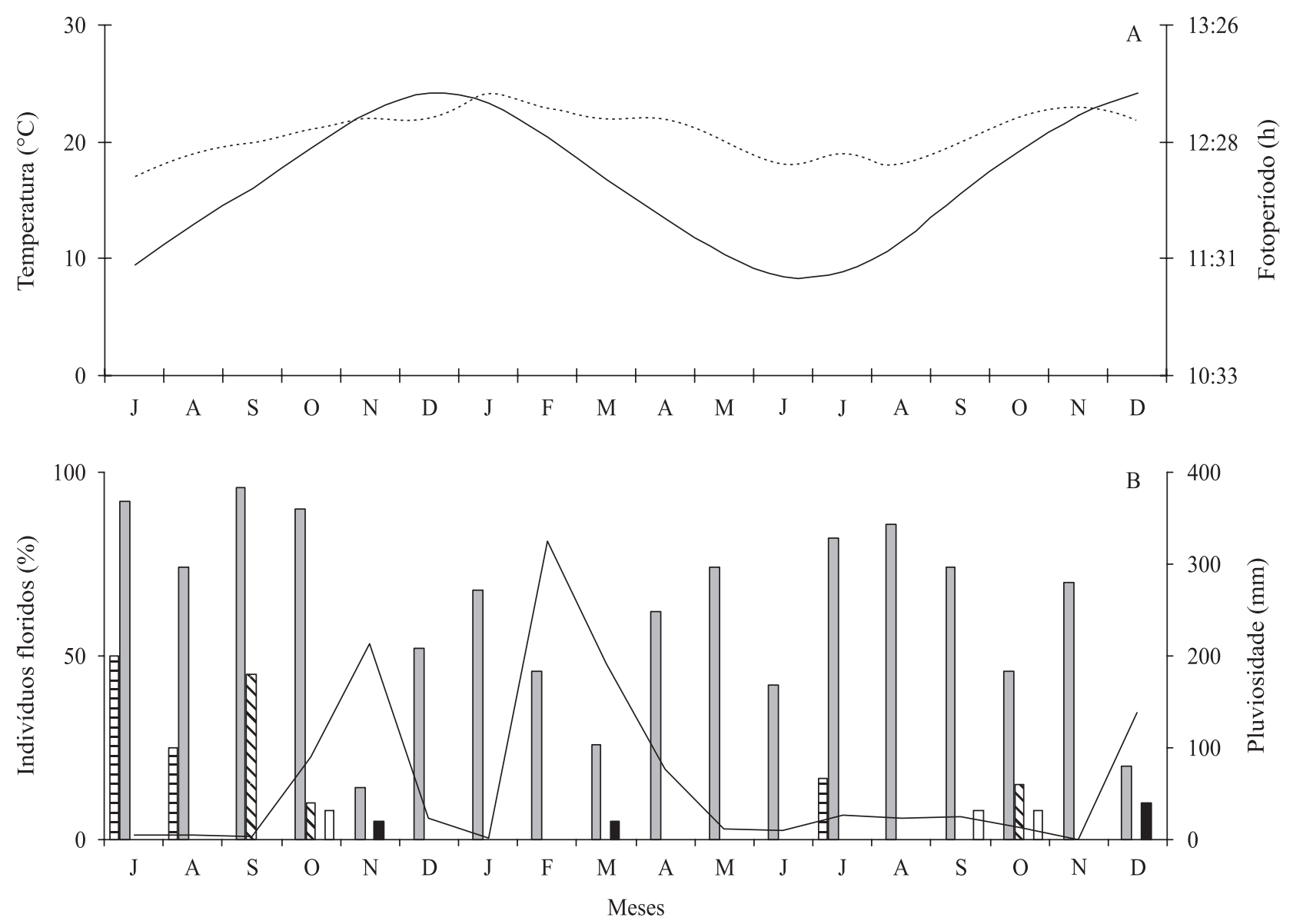

Figura 2. Dados climáticos e fenológicos de indivíduos floridos de bromeliáceas ornitófilas, no Parque Municipal de Mucugê, Mucugê, na Chapada Diamantina, Bahia, no período de julho de 2006 a dezembro de 2007. A. Temperatura $\left({ }^{\circ} \mathrm{C}\right)$ e fotoperíodo (h). B. Pluviosidade ( $\mathrm{mm}$ - linha) e frequência relativa (\% - barras).

Figure 2. Climatic and phenological data of ornitophilous flowering bromeliad individuals. Mucugê municipal park, Mucugê, Chapada Diamantina, Bahia, from July 2006 to December 2007. A. Temperature $\left({ }^{\circ} \mathrm{C}\right)$ and photoperiod (h); B. Rainfall $(\mathrm{mm}-$ line $)$ and relative frequency $(\%$ - bars $)$.

(㔷=Aechmea bromeliifolia, $n=12 ; \square=$ Hohenbergia ramageana, $n=50 ; \otimes=$ Neoregelia bahiana, $n=26 ; \boldsymbol{\square}=$ Orthophytum albopictum, $n=50 ; \square=$ Orthophytum mucugense, $n=50$ ). 
$\left.P>0,05 ; \mathrm{r}_{\mathrm{S}}=-0,011, P>0,05\right)$. As correlações entre comprimento da corola com o tamanho dos bicos dos beija-flores visitantes e com a concentração do néctar foram positivas $\left(\mathrm{r}_{\mathrm{S}}=0,476, P<0,05\right.$ e $\mathrm{r}_{\mathrm{S}}=0,501$, $P<0,05)$. Todas as espécies apresentaram néctar com concentração média de açúcares entre 20 e $30 \%$ e as suas flores encontram-se abertas durante apenas um dia, com a duração variando entre seis e 12 horas (tabela 1). Durante o período de antese em todas as espécies, o estigma esteve receptivo, bem como o pólen disponível.
Seis espécies de beija-flores (Trochilidae, Apodiformes) visitaram as flores das bromélias: Chlorostilbon lucidus (Shaw, 1812), Calliphlox amethystina (Boddaert, 1783), Chrysolampis mosquitus (Linnaeus, 1758), Augastes lumachella (Lesson, 1838), Colibri serrirostris (Vieillot, 1816) e Phaethornis pretrei (Lesson \& Delattre, 1839); também foram registradas visitas do cerebídeo (Aves: Coerebidae) Coereba flaveola (Linnaeus, 1759) (tabela 2). Eupetomena macroura (Gmelin, 1788), Thalurania glaucopis

Tabela 1: Morfologia floral das bromeliáceas ornitófilas do Parque Municipal de Mucugê, Mucugê, Chapada Diamantina, Bahia.

Table 1. Floral morphology of ornitophilous bromeliads in the Mucugê municipal park, Mucugê, Chapada Diamantina, Bahia.

\begin{tabular}{|c|c|c|c|c|c|}
\hline Espécies & $\begin{array}{l}\text { Comprimento do tubo } \\
(\mathrm{mm}) \pm \mathrm{dp}(n=10)\end{array}$ & $\begin{array}{l}\text { Concentração do néctar } \\
(\%) \pm \mathrm{dp}(n=10)\end{array}$ & $\begin{array}{l}\text { Cor da corola/ } \\
\text { anexos florais }\end{array}$ & $\begin{array}{l}\text { Período } \\
\text { de antese }\end{array}$ & $\begin{array}{l}\text { Número de } \\
\text { indivíduos }\end{array}$ \\
\hline Aechmea bromeliifolia & $11 \pm 0,3$ & $20 \pm 0,6$ & $\begin{array}{l}\text { Amarelo/brácteas } \\
\text { vermelhas }\end{array}$ & $05 \mathrm{~h} 30-11 \mathrm{~h} 30$ & 8 \\
\hline Hohenbergia ramageana & $6,3 \pm 0,3$ & $26,9 \pm 1,0$ & $\begin{array}{l}\text { Roxo/escapo } \\
\text { vermelho }\end{array}$ & $04 h 30-16 h 30$ & 35 \\
\hline Neoregelia bahiana & $45,8 \pm 3,3$ & $27,6 \pm 1,0$ & Roxo & $05 \mathrm{~h} 30-14 \mathrm{~h} 30$ & 19 \\
\hline Orthophytum albopictum & $12,2 \pm 0,4$ & $25,2 \pm 0,9$ & $\begin{array}{l}\text { Branco/folhas } \\
\text { vermelhas }\end{array}$ & $04 \mathrm{~h} 00-16 \mathrm{~h} 00$ & 27 \\
\hline Orthophytum mucugense & $9,7 \pm 0,2$ & $21,7 \pm 1$ & $\begin{array}{l}\text { Branco/folhas } \\
\text { vermelhas }\end{array}$ & $04 h 30-16 h 30$ & 58 \\
\hline
\end{tabular}

Tabela 2. Visitantes florais das bromeliáceas ornitófilas do Parque Municipal de Mucugê, Mucugê, Bahia. $(\mathrm{Cl}=$ Chlorostilbon lucidus; $\mathrm{Al}=$ Augastes lumachella $; \mathrm{Ca}=$ Calliphlox amethystina $; \mathrm{Cm}=$ Chrysolampis mosquitus; $\mathrm{Cs}=$ Colibri serrirostris; $\mathrm{Pp}=$ Phaethornis pretrei $\mathrm{Cf}=$ Coereba flaveola $; \mathrm{Am}=$ Apis mellifera $; \mathrm{Bb}=$ Bombus brevivillus; $\mathrm{Ts}=$ Trigona spinipes; $-=$ sem registro).

Table 2. Floral visitors of ornitophilous bromeliads in the Mucugê municipal park, Mucugê, Bahia. $(\mathrm{Cl}=$ Chlorostilbon lucidus; $\mathrm{Al}=$ Augastes lumachella $; \mathrm{Ca}=$ Calliphlox amethystina $; \mathrm{Cm}=$ Chrysolampis mosquitus; $\mathrm{Cs}=$ Colibri serrirostris; $\mathrm{Pp}=$ Phaethornis pretrei $\mathrm{Cf}=$ Coereba flaveola $; \mathrm{Am}=$ Apis mellifera $; \mathrm{Bb}=$ Bombus brevivillus $; \mathrm{Ts}=$ Trigona spinipes $;-=$ not registered).

\begin{tabular}{|c|c|c|c|c|c|c|c|c|c|c|c|c|}
\hline \multirow{2}{*}{ Espécies } & \multicolumn{10}{|c|}{ Visitantes } & \multirow{2}{*}{$\begin{array}{c}\text { Total de } \\
\text { visitas }\end{array}$} & \multirow{2}{*}{$\begin{array}{l}\text { Horas de } \\
\text { observação }\end{array}$} \\
\hline & $\mathrm{Cl}$ & $\mathrm{Al}$ & $\mathrm{Ca}$ & $\mathrm{Cm}$ & Cs & $\mathrm{Pp}$ & $\mathrm{Cf}$ & $\mathrm{Am}$ & $\mathrm{Bb}$ & Ts & & \\
\hline Aechmea bromeliifolia & - & - & - & - & - & 2 & 25 & 1 & - & - & 28 & 34 \\
\hline Hohenbergia ramageana & 140 & 19 & 2 & 28 & 11 & 13 & 13 & - & 2 & 5 & 233 & 272 \\
\hline Neoregelia bahiana & - & - & - & - & - & 38 & - & - & - & - & 38 & 130 \\
\hline Orthophytum albopictum & 48 & - & - & - & 1 & 5 & 6 & - & - & - & 60 & 85 \\
\hline Orthophytum mucugense & 191 & - & - & - & - & - & 166 & - & - & - & 357 & 284 \\
\hline Total de visitas & 379 & 19 & 2 & 28 & 12 & 58 & 210 & 1 & 2 & 5 & 716 & - \\
\hline Total de horas & - & - & - & - & - & - & - & - & - & - & - & 805 \\
\hline
\end{tabular}


(Gmelin, 1788), Amazilia versicolor (Vieillot, 1818), A. fimbriata (Gmelin, 1788), A. lactea (Lesson, 1832) e Anthracothorax nigricollis (Vieillot, 1817) também ocorreram na área de estudo (Machado et al. 2007), porém não visitaram flores de bromélias.

Todos os beija-flores registrados efetuaram visitas legítimas às flores, não sendo observadas tentativas de perfurações na corola para pilhagem do néctar. Coereba flaveola também realizou visitas legítimas às flores de quatro espécies de bromélias (tabela 2), no entanto este também atuou como pilhador em A. bromeliifolia. Em todas as espécies, inclusive em C. flaveola, o bico foi o local de deposição do pólen. Phaethornis pretrei visitou o maior número de espécies de bromélias, enquanto Chlorostilbon lucidus foi a espécie de beija-flor que realizou o maior número de visitas, principalmente em $O$. mucugense e $H$. ramageana (tabela 2).

As abelhas foram visitantes ocasionais, efetuando $1,12 \%$ do total das visitas registradas às flores $(n=8$ visitas). Três espécies de abelhas (Apidae, Hymenoptera) visitaram as flores das bromeliáceas: Apis mellifera realizou uma visita em $A$. bromeliifolia, enquanto Bombus brevivillus e Trigona spinipes visitaram apenas H. ramageana, somando oito visitas (tabela 2).

\section{Discussão}

As bromeliáceas ornitófilas do PMM floresceram em períodos semelhantes nos dois anos de estudo. No entanto, em outro estudo realizado na mesma área foi registrado um período de floração diferente para Aechmea bromeliifolia e Neoregelia bahiana (Machado et al. 2007). Essa diferença no padrão de floração dessas espécies pode ser decorrente de um regime pluviométrico atípico referido pelos autores, uma vez que plantas podem apresentar alterações fisiológicas como resposta à disponibilidade hídrica (Grichko \& Glick 2001).

O padrão de floração sequencial das bromélias estudadas no PMM foi registrado em outros estudos de Bromeliaceae (Araujo et al. 1994, Buzato et al. 2000, Machado \& Semir 2006). Segundo Poulin et al. (1992), isso garante a permanência dos seus polinizadores ao longo do ano, assegurando que os primeiros indivíduos de uma espécie, ao florirem, terão seu polinizador disponível. Esse padrão fenológico ocorreu em outras 13 espécies de plantas exploradas por beija-flores (Machado et al. 2007); estes autores destacaram a importância de H. ramageana para a troquilofauna local.

A floração de bromeliáceas durante a estação chuvosa tem sido registrada em diferentes ambientes
(Kaehler et al. 2005, Machado \& Semir 2006, Marques \& Lemos Filho 2008), o que parece ser comum entre plantas ornitófilas (Sazima et al. 1995). Entretanto, foi no período que antecede a estação chuvosa que houve uma maior sincronia de floração das bromeliáceas estudadas, indicando a provável frutificação durante a estação chuvosa, conforme aponta Marques \& Lemos Filho (2008). Os dados de Aechmea bromeliifolia e Hohenbergia ramageana relacionados ao fotoperíodo e à temperatura indicam que possa estar ocorrendo esta estratégia. Resultado semelhante quanto ao fotoperíodo também foi encontrado em espécies de florestas secas (Rivera \& Borchert 2001).

A concentração do néctar encontrada nas bromeliáceas estudadas é comum em plantas ornitófilas (Faegri \& van der Pijl 1979). O sistema língua/bico dos beija-flores funciona por capilaridade (Sick 1997), deste modo, quanto mais diluído for o néctar, mais fácil é a sua captação e, consequentemente, menos energia essas aves gastam pairando no ar enquanto se alimentam (Feinsinger \& Cowell 1978). Por outro lado, a baixa concentração de energia no néctar faz com que os beijaflores necessitem procurar novas flores em busca de néctar e, desta forma, visitam um grande número de flores, propiciando maior probabilidade de polinização cruzada (Feinsinger \& Cowell 1978).

A transferência de pólen pode ocorrer durante poucas visitas ou até mesmo em uma única visita se os polinizadores forem eficientes (Ramirez et al. 1990). É comum em bromeliáceas ornitófilas, incluindo as aqui estudadas, flores com duração de abertura de apenas um só dia, com um período curto de antese (Araujo et al. 1994, Martinelli 1997, Machado \& Semir 2006). A garantia da polinização se dá pela liberação constante de pólen e pela grande probabilidade de visitas dos beija-flores, uma vez que estas aves, nectarívoras especializadas, são atraídas pela produção de néctar associada às características morfológicas, também especializadas (Sick 1997, Machado \& Semir 2006, Machado \& Rocca 2008, Machado 2009).

As bromeliáceas ornitófilas do PMM apresentam flores generalistas, com exceção de Neoregelia bahiana que foi visitada apenas por Phaethornis pretrei, provavelmente o único beija-flor na área capaz de explorar o néctar desta planta devido ao tamanho do seu bico e, consequentemente, efetuar a sua polinização, sugerindo uma estreita relação entre $N$. bahiana e $P$. pretrei, que teve este como seu único polinizador.

Flores com corola longa apresentam uma baixa diversidade de beija-flores polinizadores (Wolf et al. 1976), comparando com as espécies que possuem corola 
pequena. Nestes casos, espera-se que as primeiras possuam concentrações de açúcares no néctar mais altas que as das flores de corola curta para que haja uma maior recompensa aos seus polinizadores especialistas (Feinsinger 1983), tal como ocorreu neste estudo.

Em diversas investigações relacionadas a beija-flores e à comunidade de plantas que exploram, incluindo o presente estudo, constatou-se que há uma correlação positiva entre o tamanho dos bicos destas aves e o tamanho do tubo das corolas das plantas que visitam (Arizmendi \& Ornelas 1990, Vasconcelos \& Lombardi 2001). Em alguns estudos feitos na Mata Atlântica (Buzato et al. 2000, Machado \& Semir 2006) e mesmo no estudo realizado no PMM, que considerou bromeliáceas e não-bromeliáceas (Machado et al. 2007), a correlação entre estas duas variáveis, ainda que positiva, não foi significativa. Assim, como consideraram estes últimos autores, é necessário cautela em generalizações quanto a esta relação, uma vez que beija-flores de bico longo podem visitar espécies de corola curta. A tendência a um ajuste entre o tamanho do aparelho bucal à profundidade da corola de flores visitadas não é apenas restrito às aves, mas ocorre também em algumas guildas de insetos polinizadores de diferentes plantas (Cogliatti-Carvalho \& Rocha 2004).

Assim como reportado para outras espécies da subfamília Bromelioideae (Araujo et al. 2004), o pólen das bromeliáceas estudadas foi depositado nos bicos dos beija-flores. Há registros de comunidades onde o pólen é depositado no mesmo local do corpo dos vetores e o padrão de floração sequencial divide temporalmente o uso do polinizador (Araujo et al. 1994, Machado \& Semir 2006).

No PMM, Phaethornis pretrei não permaneceu próximo às flores que visitou. Os fetornitíneos tendem a ser mais especializados no uso de tipos florais, uma vez que possuem bicos longos e mais curvos, o que não impede que visitem flores de corolas curtas, forrageando preferencialmente recursos isolados e estabelecendo linhas de captura (Feinsinger \& Colwell 1978). Como conseqüência, há maiores chances de polinização cruzada.

As bromeliáceas frequentemente produzem abundante mucilagem quando injuriadas; também deve-se considerar que flores encerradas dentro da roseta (como em Orthophytum albopictum, O. mucugense e Neoregelia bahiana) ou densamente agrupadas na inflorescência (Aechmea bromeliifolia) podem inviabilizar a pilhagem de néctar, tanto de Coereba flaveola quanto de beijaflores, através de orifícios laterais na corola.
Em Bromeliaceae a polinização por insetos não é comum e, quando ocorre, é realizada por abelhas (Alves et al. 2000, Araujo et al. 2004) ou borboletas (Alves et al. 2000, Varassin \& Sazima 2000, Kaehler et al. 2005). Dentre as bromeliáceas estudadas, H. ramageana parece ser a mais generalista quanto a seus visitantes, devido à sua curta corola. A coloração azulada de suas flores pode ser atrativa para insetos, sobretudo abelhas. Entretanto, a freqüência de visitas de insetos às flores desta bromélia foi insignificante diante das efetuadas pelos beija-flores, sobretudo Chlorostilbon lucidus, considerado como seu principal polinizador no PMM. Todavia as abelhas podem ser consideradas polinizadoras de $H$. ramageana e $A$. bromeliifolia, pois os órgãos sexuais das plantas foram contatados durante o forrageio, além de permanecerem nas flores por mais tempo que os beija-flores.

As bromélias ornitófilas do PMM aqui estudadas, a maioria endêmica da Chapada Diamantina, são importantes para a manutenção da fauna de beija-flores residentes desta área de campo rupestre, o que beneficia diretamente outras espécies de plantas que utilizam estas aves como vetores de pólen. Estudos semelhantes conduzidos em outras áreas da Cadeia do Espinhaço são indispensáveis para subsidiar o entendimento dos padrões de distribuição e evolução de bromélias ornitófilas e seus beija-flores nesta formação e, conseqüentemente, possibilitar planos de políticas conservacionistas de suas espécies e comunidades.

Agradecimentos - Os autores agradecem ao Programa de Pós Graduação em Botânica (PPGBot/UEFS), ao qual este trabalho está vinculado; à Coordenação de Aperfeiçoamento de Pessoal de Nível Superior (Capes), pela bolsa concedida a C.S. Santana; à Prefeitura Municipal de Mucugê, pela autorização deste estudo no Parque Municipal de Mucugê e por todo o apoio logístico; à Universidade Estadual de Feira de Santana (UEFS) e ao Laboratório de Ornitologia (Ornito) e sua equipe, pela infra-estrutura disponibilizada e auxílios; aos Drs. F.F. Oliveira, pela identificação das abelhas, L.P. Queiroz e A.A. Conceição, pela identificação das bromélias; e às MSc. A.M. Alves e A.G. Coelho pela ajuda em campo.

\section{Referências bibliográficas}

ALTMANN, S.A. 1974. Observational study of behavior: sampling methods. Behavior 49:227-265.

ALVES, A.S.A., SILVA, J.M.C., VAN SLUYS, M., BERGALLO, H.G. \& ROCHA, C.F.D. 2000. A ornitologia no Brasil. Universidade Estadual do Rio de Janeiro - UERJ, Rio de Janeiro. 
ARAUJO, A.C., FISCHER, E.A. \& SAZIMA, M. 1994. Floração sequencial e polinização de três espécies de Vriesea (Bromeliaceae) na região de Juréia, sudeste do Brasil. Revista Brasileira de Botânica 17:113-118.

ARAUJO, A.C., FISCHER, E.A. \& SAZIMA, M. 2004. As bromélias na região do Rio Verde. In Estação Ecológica Juréia-Itatins. Ambiente físico, flora e fauna (O.A.V. Marques \& W. Duleba, eds.). Holos Editora, São Paulo, p.162-171.

ARIZMENDI, M.C. \& ORNELAS, J.F. 1990. Hummingbirds and their floral resources in tropical dry forest in Mexico. Biotropica 22:172-180.

BENCKE, C.S.C. \& MORELLATO, L.P.C. 2002. Comparação de dois métodos de avaliação da fenologia de plantas, sua interpretação e representação. Revista Brasileira de Botânica 25:269-275.

BENZING, D.H. 1980. The biology of the bromeliads. Mad River Press, Eureka.

BUZATO, S., SAZIMA, M. \& SAZIMA, I. 2000. Hummingbird-pollinated floras at three Atlantic Forest sites. Biotropica 32:824-841.

CBRO - Comitê Brasileiro de Registros Ornitológicos. 2007. Lista das aves do Brasil. Sociedade Brasileira de Ornitologia. http://www.cbro.org.br (acesso em 07/04/2008).

COGLIATTI-CARVALHO, L. \& ROCHA, C.F.D. 2004. Relação entre a profundidade da corola e comprimento do aparelho bucal dos visitantes florais de sete espécies vegetais em uma área de Mata Atlântica da Ilha Grande, RJ. In Anais do VI Simpósio de Ecossistemas Brasileiros. Aciesp, São Paulo, v.2, p.342-350.

CONCEIÇÃO, A.A. \& GIULIETTI, A.M. 2002. Composição florística e aspectos estruturais de campo rupestre em dois platôs do Morro do Pai Inácio, Chapada Diamantina, Bahia, Brasil. Hoehnea 29:37-48.

CONCEIÇÃO, A.A. \& PIRANI, J.R. 2005. Delimitação de habitats em campos rupestres na Chapada Diamantina, Bahia: substratos, composição florística e aspectos estruturais. Boletim de Botânica da Universidade de São Paulo 23:85-111.

DAFNI, A. 1992. Pollination ecology - A practical approach. Oxford University Press, Oxford.

FAEGRI, K. \& VAN DER PIJL, L. 1979. The principles of pollination ecology. Pergamon Press, New York.

FAUSTINO, T.C. \& MACHADO, C.G. 2006. Frugivoria por aves em uma área de campo rupestre na Chapada Diamantina, BA. Revista Brasileira de Ornitologia 14:137-143.

FEINSINGER, P. 1983. Variable nectar secretion in a Heliconia species pollinated by hermit hummingbirds. Biotropica 15:48-52.

FEINSINGER, P. \& COWELL, R.K. 1978. Community organization among neotropical nectar feeding birds. American Zoologist 18:779-795.
FRAGA, R.M. 1989. Interations between nectarivorous birds and the flowers of Aphelandra sinclairiana in Panama. Journal of Tropical Ecology 5:19-26.

GIULIETTI, A.M., PIRANI, J.R. \& HARLEY, R.M. 1997. Espinhaço Range region, Eastern Brazil. In Centers of plant diversity, a guide and strategy for their conservation 3. (S.D. Davis, V.H. Heywood, O. H. MacBryde, J. Villalobos \& A.C. Hamilton, eds.). Information Press, Oxford.

GRANTSAU, R. 1988. Os beija-flores do Brasil. Expressão e Cultura, Rio de Janeiro.

GRICHKO, V.P. \& GLICK, B.R. 2001. Ethylene and flooding stress in plants. Plant Physiology and Biochemistry 39:1-9.

HARLEY, R.M. \& SIMMONS, N.A. 1986. Florula of Mucugê - Chapada Diamantina - Bahia, Brazil. Royal Botanic Gardens, Kew.

KAEHLER, M., VARASSIN, I.G. \& GOLDENBERG, R. 2005. Polinização em uma comunidade de bromélias em Floresta Atlântica Alto-montana no Estado do Paraná, Brasil. Revista Brasileira de Botânica 28:219-228.

LAMMI, J. 2001. On line photoperiod calculator. http://www. sci.fi/ benefon/sol.html (acesso em 07/04/2008).

LEAL, F.C., LOPES, A.V. \& MACHADO, I.C. 2006. Polinização por beija-flores em uma área de caatinga no município de Floresta, Pernambuco, Nordeste do Brasil. Revista Brasileira de Botânica 29:379-389.

LUTHER, H.E. 2004. An alphabetical list of bromeliad binomials. The Bromeliad Society International, Orlando.

MACHADO, C.G. 2009. Beija-flores (Aves: Trochilidae) e seus recursos florais em uma área de caatinga da Chapada Diamantina, Bahia, Brasil. Zoologia 26:255-265.

MACHADO, C.G. \& ROCCA, M.A. 2008. Protocolos para o estudo da polinização por aves. In Ornitologia e Conservação: Ciência Aplicada, Técnicas de Pesquisa e Levantamento (S.V. Matter, F.C. Straube, I. Accordi, V. Piacentini \& J.F. Cândido-Jr., eds). Technical Books, Rio de Janeiro, p.471-489.

MACHADO, C.G. \& SEMIR, J. 2006. Fenologia da floração e biologia floral de bromeliáceas ornitófilas de uma área da Mata Atlântica do Sudeste brasileiro. Revista Brasileira de Botânica 29:163-174.

MACHADO, C.G., COELHO, A.G., SANTANA, C.S. \& RODRIGUES, M. 2007. Beija-flores e seus recursos florais em uma área de campo rupestre da Chapada Diamantina, Bahia. Revista Brasileira de Ornitologia 15:215-227.

MARQUES, A.R. \& LEMOS FILHO, J.P. 2008. Fenologia reprodutiva de espécies de bromélias na Serra da Piedade, MG, Brasil. Acta Botanica Brasilica 22:417-424.

MARTINELLI, G. 1997. Biologia reprodutiva de Bromeliaceae na Reserva Ecológica de Macaé de Cima. In Serra de Macaé de Cima: Diversidade florística e conservação em Mata Atlântica (H.C. Lima \& R.R. Guedes-Bruni, eds). Jardim Botânico do Rio de Janeiro, Rio de Janeiro, p.213-250. 
MORELLATO, L.P.C. \& LEITÃO-FILHO, H.F. 1992. Padrões de frutificação e dispersão na Serra do Japi. In História natural da Serra do Japi: ecologia e preservação de uma área florestal no Sudeste do Brasil (L.P.C. Morellato, org.). Editora da Universidade Estadual de Campinas - Unicamp e Fundação de Amparo à Pesquisa do Estado de São Paulo - Fapesp, Campinas, p.112-140.

NEWSTRON, L.E., FRANKIE, G.W. \& BAKER, H.G. 1994. A new classification of plant phenology basead on flowering patterns in lowlands tropical rain forest trees at la Selva, Costa Rica. Biotropica 26:141-159.

PIACENTINI, V.Q. \& VARASSIN, I.G. 2007. Interaction network and the relationships between bromeliads and hummingbirds in an area of secondary Atlantic rain forest in southern Brazil. Journal of Tropical Ecology 23:663-671.

POULIN, B., LEFEBVRE, G. \& MCNEIL, R. 1992. Tropical avian phenology in relation to abundance and exploitation of food resources. Ecology 73: 2295-2309.

RAMIREZ, N., GIL, C., HOKCHE, O., SERES, A. \& BRITO, Y. 1990. Biologia floral de una comunidad arbustiva tropical en la Guayana Venezolana. Annals of the Missouri Botanical Garden 77:383-397.

REITZ, R. 1983. Bromeliáceas e a malária - bromélia endêmica. Flora Ilustrada Catarinense (R. Reitz, ed.). Herbário Barbosa Rodrigues, Itajaí, p.1-559.

RIVERA, G. \& BORCHERT, R. 2001. Induction of flowering in tropical trees by a 30 -min reduction in photoperiod: evidence from field observations and herbarium collections. Tree Physiology 21:201-212.

ROCHA, C.F.D., COGLIATTI-CARVALHO, L., NUNES-FREITAS, A.F., ROCHA-PESSOA, T.C., DIAS, A.S., ARIANI, C.V. \& MORGADO, L. 2004. Conservando uma larga porção da diversidade biológica através da conservação de bromeliácea. Vidalia 2:52-68.

SAN MARTIN-GAJARDO, I. \& MORELLATO, L.P.C. 2003. Fenologia de Rubiaceae do sub-bosque em floresta Atlântica no Sudeste do Brasil. Revista Brasileira de Botânica 26:299-309.

SANTOS, R.L., ALMEIDA, M.G., NUNES, J.V., TINOCO, L.D.S. \& MARTINS, L.B. 2003. Bromeliads as a keystone resource for the scorpion Tityus neglectus in eastern Rio Grande do Norte State. Journal of the Bromeliad Society 53:256-258.
SAZIMA, I., BUZATO, S. \& SAZIMA, M. 1995. The Saw-billed Hermit Ramphodon naevius and its flowers in southeastern Brazil. Journal of Ornithology 136: 195-206.

SICK, H. 1997. Ornitologia brasileira. Ed. Nova Fronteira. Rio de Janeiro.

SILVA, J.M.C. 1998. Integrating biogeography and conservation: an example with birds and plants of the Cerrado region. Anais da Academia Brasileira de Ciências 70:881-888.

SIQUEIRAFILHO, J.A. 1998. Biologia floral de Hohenbergia ridleyi (Baker) Mez. Bromélia 5:1-13.

SIQUEIRA FILHO, J.A. \& MACHADO, I.C.S. 2001. Biologia reprodutiva de Canistrum aurantiacum E. Morren (Bromeliaceae) em remanescentes da Floresta Atlântica, Nordeste do Brasil. Acta Botanica Brasilica 15:427-444.

SNOW, D.W. \& SNOW, B.K. 1986. Feeding ecology of hummingbirds in the Serra do Mar, southeastern Brazil. Hornero 12:286-296.

STRADMANN, M.P.S. 1998. Plano de Manejo - Parque Municipal de Mucugê. Projeto Sempre-Viva / MMA/ PNMA/PED 96CV00027/96, Mucugê.

TALORA, D.C. \& MORELLATO, P.C. 2000. Fenologia de espécies arbóreas em floresta de planície litorânea do sudeste do Brasil. Revista Brasileira de Botânica 23:13-26.

VARASSIN, I.G. \& SAZIMA, M. 2000. Recursos de Bromeliaceae utilizados por beija-flores e borboletas em Mata Atlântica no Sudeste do Brasil. Boletim do Museu de Biologia Mello Leitão 11/12:57-70.

VASCONCELOS, M.F. \& LOMBARDI, J.A. 2001. Hummingbirds and their flowers in the campos rupestres of Southern Espinhaço Range, Brazil. Melopsittacus 4:3-30.

VERSIEUX, L.M. \& WENDT, T. 2007. Bromeliaceae diversity and conservation in Minas Gerais state, Brazil. Biodiversity and Conservation 16:2989-3009.

WANDERLEY, M.G.L. \& CONCEIÇÃO, A.A. 2006. Notas taxonômicas e uma nova espécie do gênero Orthophytum Beer (Bromeliaceae) da Chapada Diamantina, Bahia, Brasil. Sitientibus, Série Ciências Biológicas 6:3-8.

WOLF, L.L., STILES, F.G. \& HAINSWORTH, F.R. 1976. Ecological organization community. Journal of Animal Ecology 32:149-179.

ZAR, J.H. 1996. Bioestatistical analysis. Prentice-Hall, New Jersey. 